\title{
Cardiac involvement in COVID-19 patients: risk factors, predictors, and complications: A Review
}

\author{
Ghazal Aghagoli et al. ${ }^{1}$ \\ ${ }^{1}$ Affiliation not available
}

April 28, 2020

\begin{abstract}
Respiratory complications have been well remarked in the novel coronavirus disease (SARS-CoV-2/COVID-19), yet an emerging body of research indicates that cardiac involvement may be implicated in poor outcomes for these patients. This review seeks to gather and distill the existing body of literature that describes the cardiac implications of COVID-19. Notably, COVID-19 patients with pre-existing cardiovascular disease are counted in greater frequency in intensive care unit settings, and ultimately suffer greater rates of mortality. Other studies have noted cardiac presentations for COVID-19, rather than respiratory, such as acute pericarditis and left ventricular dysfunction. In some patients there has been evidence of acute myocardial injury, with correspondingly increased serum troponin I levels. With regard to surgical interventions, there is a dearth of data describing myocardial protection during cardiac surgery for COVID-19 patients. Although some insights have been garnered in the study of cardiovascular diseases for these patients, these insights remain fragmented and have yet to cement clear guidelines for actionable clinical practice. Further studies are imperative for a more cohesive understanding of the cardiac pathophysiology in COVID-19 patients to promote more informed treatment and, ultimately, better clinical outcomes.
\end{abstract}

Published in Journal of Cardiac Surgery. DOI forthcoming.

\section{Full author list}

Ghazal Aghagoli ${ }^{1}$, BS, Benjamin Gallo Marin, AB ${ }^{1}$, Luke B. Soliman, MTS ${ }^{1}$, Frank W. Sellke, MD ${ }^{1,2}$

${ }^{1}$ Warren Alpert Medical School of Brown University, Providence, Rhode Island, United States

${ }^{2}$ Division of Cardiothoracic Surgery, Rhode Island Hospital, Providence, Rhode Island, United States

\section{Funding Sources}

This research was supported in part by NIH Grant's R01 HL46716 and R01HL 128831.

\section{Introduction}

Since the initial outbreak of the novel coronavirus disease (SARS-CoV-2/COVID-19) in December, 2019 (from Wuhan, China), there have been 693,282 confirmed cases and 33,106 deaths worldwide as of March, $30,2020{ }^{1,2}$ Symptomatic patients usually present with fever, dry cough, and shortness of breath, which may appear 2-14 days after infection. ${ }^{3}$ While it has been demonstrated that this virus has a predilection for the lungs and that respiratory complications are strongly associated with mortality, emerging reports show that cardiac involvement can be present in COVID-19 patients. Here, we synthesize the literature to describe the 
various cardiac findings in COVID-19 that have been made in relation to risk factors, predictors of progression, and complications during and post COVID-19 infection. We aim to provide a comprehensive review on this matter to assist physicians and researchers in their efforts to efficiently update their understanding to better address the various burdens of the current global pandemic.

Cardiac risk factors have been identified that predict the susceptibility to COVID-19 infection and illness severity. According to the Centers for Disease Control and Prevention (CDC), elderly patients with comorbidities at a higher risk to become infected with COVID-19, especially those with coronary heart disease, hypertension, or diabetes. ${ }^{4}$ Cardiovascular diseases are also associated with worse prognosis and more severe progression of COVID-19. A study that investigated infected patients who received care in the intensive care unit (ICU) reported the frequency of cardio-cerebrovascular diseases, hypertension, and diabetes to be 3 folds, 2 folds, and 2 folds, respectively, higher than counterparts receiving non-ICU care. ${ }^{5} \mathrm{~A}$ different investigation focusing on patients with severe symptoms described that $25 \%$ had heart diseases, $44 \%$ had arrhythmia, and 58\% had hypertension. ${ }^{6}$ Cardiac injury has been associated with COVID-19 mortality as well. One study found that patients with cardiac injury had higher mortality than those without (51.2\% vs. $4.5 \%$, respectively). ${ }^{7}$ In this same study, Cox regression model showed that patients with cardiac injury were at a higher risk of death both from time of symptom onset (hazard ratio, 4.26 [95\% CI 1.92-9.49]) and from the time of hospital admission to end point (hazard ratio, 3.41 [95\% CI, 1.62-7.16]). ${ }^{7}$ Taken together, there is mounting evidence that underlying cardiovascular conditions lead to higher likelihood of infection, more severe disease progression, and greater risk for mortality from COVID-19.

Interestingly, recent evidence suggests that cardiac signs can be useful predicting factors in distinguishing mild versus severe COVID-19 disease progression. While the virus does have a predilection for the lungs, the infection also involves damage to the heart, vessels, liver, kidney, and other organs. ${ }^{8}$ This suggests that there may be pathological signs pertaining to organ systems other than lungs that could be relevant in the generation of a reliable prognosis. Indeed, it has been found that Troponin I levels are only marginally increased in all patients with COVID-19, but values exceeding the 99th percentile in the upper reference limit are only observed in 8-12\% of positive cases. ${ }^{9}$ Early measurement of biomarkers for cardiac damage upon a suspected infected patient's admission to the hospital could help identify cardiac injury, which ultimately predicts a more severe prognosis.

Cardiac injury in COVID-19 may result from direct effects of the virus itself. In general, viral infections are one of the most common causes of infectious myocarditis. ${ }^{10}$ Evidence also suggests that common infections trigger acute coronary events and strokes. ${ }^{11,12}$ Following this vein, researchers have aimed to describe the mechanisms of COVID-19-mediated cardiac injury. The virus infects host cells through angiotensinconverting enzyme-2 (ACE2) receptors which can lead to pneumonia, acute myocardial injury, and chronic cardiovascular damage. ${ }^{13} \mathrm{ACE} 2$ receptors, which are important in the cardiovascular and immune systems, are membrane bound aminopeptidases. These receptors are highly expressed in the heart and lungs, and they have been confirmed to be the functional receptors for the novel coronavirus. ${ }^{14}$ These findings indicate that myocardial injury caused in COVID-19 might be ACE2 related. Given this mechanism of action, there has been substantial discussion and controversy on the use of antihypertensive ACE inhibitors in COVID-19 infected patients. Updates from the AHA suggest that, based on mortality data, ACE inhibitors should be maintained or initiated in patients with myocardial infections, heart failure, or hypertension. ${ }^{15}$ Multiple review articles have echoed this guideline, indicating that withdrawal of RAAS inhibitors in patients with COVID-19 may be harmful. ${ }^{16,17}$

Recent studies and case reports have shed light on the potential of acute cardiac injury occurring in COVID-19 infected patients. Middle east respiratory syndrome related coronavirus (MERS-CoV) can cause myocarditis and heart failure. ${ }^{18}$ Both COVID-19 and MERS-CoV have relatively similar pathogenicity, with myocardial damage being associated in both diseases, leading to increased complexity in patient treatment. ${ }^{13}$ One case report discussed a COVID-19 patient with left ventricular dysfunction and acute pericarditis who presented without any respiratory tract signs or symptoms. ${ }^{19}$ An investigation of the clinical features of patients with COVID-19 found myocardial injury in 5 out of 41 patients, and it mainly manifested as an increase in 
high sensitivity cardiac troponin 1 levels. ${ }^{20}$ Importantly, cardiac involvement has not only been present in adults but shown to be present in infants as well. A case of a 55 day old infant who presented with pneumonia, liver injury, and myocardial injury has been reported in China. ${ }^{21}$ This pediatric patient had an abnormal myocardial zymogram on admission, occasional tachycardia (150-170 bpm) on hospital days 2-6, and increased troponin I levels $\left(0.025\right.$ microgram/L) on hospital day $4 .^{21}$ These findings suggest that myocarditis as a complication of COVID-19 should be considered and might help improve management and treatment of infected patients.

Cardiac issues in COVID-19 may also be related to the pharmacologic interventions. Antiviral medications are a common treatment, and an investigation of 138 COVID-19 infected patients reported that $89.9 \%$ of patients were given such medications. ${ }^{6}$ It has been previously established that antiviral drugs can cause cardiac insufficiency, arrhythmias, and other cardiovascular disorders. ${ }^{22}$ Therefore, cardiac injuries caused by pharmacological treatments must be kept in mind while managing COVID-19 infected patients.

The question of whether chronic cardiac issues are present post COVID-19 recovery still remains. Although much about this question is currently not well known, studies from chronic cardiac outcomes of SARS-CoV, which is structurally similar to SARS-CoV-2, provides evidence that these outcomes are important considerations. A longitudinal study following patients that recovered from SARS-CoV reported that $68 \%$ had hyperlipidemia, $4 \%$ had cardiovascular system abnormalities, and $60 \%$ had glucose metabolism disorders post recovery. ${ }^{23}$ Furthermore, lipid metabolism dysregulation in patients with a history of SARS-CoV infection and serum concentration of free fatty acids, lysophosphatidylcholine, lysophosphatidylethanolamine, and phosphatidylglycerol were significantly increased compared with people without history of SARS-CoV infection. ${ }^{23}$ These results suggest that SARS-CoV is associated with cardiovascular diseases and serum metabolic alterations. Based on these findings, chronic cardiac diseases as a coronavirus-associated pathology must be considered and further investigations of cardiac conditions post COVID-19 are required.

When do the pathology processes of the COVID-19 affect patient care, in addition to an obvious detrimental effect on myocardial function leading to heart failure? If a patient presents with underlying congestive heart failure, COVID-19 can markedly increase the severity of the illness. Furthermore, COVID-19 does not preclude coronary artery plaque rupture and myocardial infarction or progression of coronary artery disease. Patients needing urgent coronary artery bypass grafting, aortic valve replacement, repair of an aortic dissection, other urgent or emergent operation may be more prone to myocardial dysfunction after the surgery, especially if cardioplegic arrest is used. There is no data on the effect of COVID-19 on myocardial protection during cardiac surgery. Patients undergoing percutaneous coronary intervention (PCI) or coronary stenting, may have a similar poor outcome due to the additive effect of myocardial ischemia and poor underlying myocardial function.

\section{Conclusion}

The effect of COVID-19 on myocardial function are not well established, nor are its effects on the outcomes after treatment of myocardial and associated illnesses. Based on what has been discovered and hypothesized about cardiac involvement in COVID-19, there is a need for autopsies and rigorous gross, histological cardiac assessments, and more basic research into the effects of COVID-19 on myocardial function and other associated clinical conditions such as diabetes. Such analyses could further elucidate the mechanisms that seem to be at play in COVID-19-mediated heart dysregulation and injury. In addition, we recommend researchers to consider how imaging methods may be used to detect abnormalities in heart function in infected patients, as well as further scrutiny of cardiac blood chemistry. These findings will ultimately assist providers in detecting clinicopathological signs as early as possible that may help predict disease severity and better inform treatment management.

\section{Disclosures}

The authors have nothing to disclose.

\section{Author Contributions}


G Aghagoli and B Gallo Marin developed the early ideas of this paper. G Aghagoli, B Gallo Marin, L Soliman, and F Sellke were the main writers of the initial draft. All authors contributed equally to the editing of the final manuscript.

\section{References}

1. Zhu N, Zhang D, Wang W, Li X, Yang B, Song J, Zhao X, Huang B, Shi W, Lu R, Niu P, Zhan F, Ma X, Wang D, Xu W, Wu G, Gao GF, Tan W. A Novel Coronavirus from Patients with Pneumonia in China, 2019. N Engl J Med 2020;382:727-733.

2. World Health Organization . Coronavirus disease 2019 ( COVID-19): situation report, 70. 2020. https://www.who.int/docs/default-source/coronaviruse/situation-reports/20200330-sitrep-70-covid-19.pdf?sfvrsn=7e0fe3f8_4 .

3. Centers for Disease Control and Prevention. Coronavirus Disease 2019 (COVID-19) - Symptoms. https://www.cdc.gov/coronavirus/2019-ncov/symptoms-testing/symptoms.html. Accessed March 26, 2020.

4. Centers for Disease Control and Prevention. Coronavirus Disease 2019 (COVID-19). https://www.cdc.gov/coronavirus/201 ncov/need-extra-precautions/people-at-higher-risk.html?CDC_AA_refVal=https\%3A\%2F\%2Fwww.cdc.gov\%2Fcoronavirus\% ncov\%2Fspecific-groups\%2Fhigh-risk-complications.html. Accessed March 31, 2020.

5. Li B, Yang J, Zhao F, Zhi L, Wang X, Liu L, Bi Z, Zhao Y. Prevalence and impact of cardiovascular metabolic diseases on COVID-19 in China. Clin Res Cardiol 2020.

6. Wang D, Hu B, Hu C, Zhu F, Liu X, Zhang J, Wang B, Xiang H, Cheng Z, Xiong Y, Zhao Y, Li Y, Wang X, Peng Z. Clinical Characteristics of 138 Hospitalized Patients With 2019 Novel Coronavirus-Infected Pneumonia in Wuhan, China. JAMA 2020.

7. Shi S, Qin M, Shen B, Cai Y, Liu T, Yang F, Gong W, Liu X, Liang J, Zhao Q, Huang H, Yang B, Huang C. Association of Cardiac Injury With Mortality in Hospitalized Patients With COVID-19 in Wuhan, China. JAMA Cardiol 2020.

8. Yao XH, Li TY, He ZC, Ping YF, Liu HW, Yu SC, Mou HM, Wang LH, Zhang HR, Fu WJ, Luo T, Liu F, Chen C, Xiao HL, Guo HT, Lin S, Xiang DF, Shi Y, Li QR, Huang X, Cui Y, Li XZ, Tang W, Pan PF, Huang XQ, Ding YQ, Bian XW. A pathological report of three COVID-19 cases by minimally invasive autopsies. Zhonghua Bing Li Xue Za Zhi 2020;49:E009.

9. Lippi G, Plebani M. Laboratory abnormalities in patients with COVID-2019 infection. Clin Chem Lab Med 2020.

10. Fung G, Luo H, Qiu Y, Yang D, McManus B. Myocarditis. Circ Res 2016;118:496-514.

11. Elkind MSV. Why now? Moving from stroke risk factors to stroke triggers. Curr Opin Neurol 2007;20:5157.

12. Smeeth L, Thomas SL, Hall AJ, Hubbard R, Farrington P, Vallance P. Risk of myocardial infarction and stroke after acute infection or vaccination. N Engl J Med 2004;351:2611-2618.

13. Zheng Y-Y, Ma Y-T, Zhang J-Y, Xie X. COVID-19 and the cardiovascular system. Nat Rev Cardiol 2020 .

14. Hoffmann M, Kleine-Weber H, Schroeder S, Krüger N, Herrler T, Erichsen S, Schiergens TS, Herrler G, Wu N-H, Nitsche A, Müller MA, Drosten C, Pöhlmann S. SARS-CoV-2 Cell Entry Depends on ACE2 and TMPRSS2 and Is Blocked by a Clinically Proven Protease Inhibitor. Cell 2020.

15. American Heart Associatin. HFSA/ACC/AHA statement addresses concerns re: using RAAS antagonists in COVID-19. https://professional.heart.org/professional/ScienceNews/UCM_505836_HFSAACCAHAstatementaddresses-concerns-re-using-RAAS-antagonists-in-COVID.jsp. Accessed March 28, 2020. 
16. Kuster GM, Pfister O, Burkard T, Zhou Q, Twerenbold R, Haaf P, Widmer AF, Osswald S. SARS-CoV2: should inhibitors of the renin-angiotensin system be withdrawn in patients with COVID-19? Eur Heart J 2020 .

17. Vaduganathan M, Vardeny O, Michel T, McMurray JJV, Pfeffer MA, Solomon SD. Renin-AngiotensinAldosterone System Inhibitors in Patients with Covid-19. N Engl J Med 2020.

18. Alhogbani T. Acute myocarditis associated with novel Middle east respiratory syndrome coronavirus. Ann Saudi Med 2016;36:78-80.

19. Inciardi RM, Lupi L, Zaccone G, Italia L, Raffo M, Tomasoni D, Cani DS, Cerini M, Farina D, Gavazzi E, Maroldi R, Adamo M, Ammirati E, Sinagra G, Lombardi CM, Metra M. Cardiac Involvement in a Patient With Coronavirus Disease 2019 (COVID-19). JAMA Cardiol 2020.

20. Huang C, Wang Y, Li X, Ren L, Zhao J, Hu Y, Zhang L, Fan G, Xu J, Gu X, Cheng Z, Yu T, Xia J, Wei Y, Wu W, Xie X, Yin W, Li H, Liu M, Xiao Y, Gao H, Guo L, Xie J, Wang G, Jiang R, Gao Z, Jin Q, Wang J, Cao B. Clinical features of patients infected with 2019 novel coronavirus in Wuhan, China. Lancet 2020;395:497-506.

21. Cui Y, Tian M, Huang D, Wang X, Huang Y, Fan L, Wang L, Chen Y, Liu W, Zhang K, Wu Y, Yang Z, Tao J, Feng J, Liu K, Ye X, Wang R, Zhang X, Zha Y. A 55-Day-Old Female Infant infected with COVID 19: presenting with pneumonia, liver injury, and heart damage. J Infect Dis 2020.

22. Sakabe M, Yoshioka R, Fujiki A. Sick sinus syndrome induced by interferon and ribavirin therapy in a patient with chronic hepatitis C. J Cardiol Cases 2013;8:173-175.

23. Wu Q, Zhou L, Sun X, Yan Z, Hu C, Wu J, Xu L, Li X, Liu H, Yin P, Li K, Zhao J, Li Y, Wang X, Li Y, Zhang Q, Xu G, Chen H. Altered Lipid Metabolism in Recovered SARS Patients Twelve Years after Infection. Sci Rep 2017;7:9110. 